\title{
A BOUND FOR SOLUTIONS OF VOLTERRA-STIELTJES INTEGRAL EQUATIONS
}

R. H. MARTIN, JR.

W. A. Coppel [1, p. 58] uses a "measure" $\mu$ defined on square matrices by $\mu[A]=\lim _{h \rightarrow+0}(|1+h A|-1) / h$ in order to obtain a bound for solutions to the linear differential equation $y^{\prime}=A y$. This paper ex tends this result to a Volterra-Stieltjes integral equation

$$
y(x)=1+\int_{x}^{z} d F \cdot y
$$

where $F$ is of bounded variation on each bounded subinterval and the integral is the Cauchy right integral. Initial value problems of this type have been investigated by J. S. MacNerney and others. See [4] for references.

Notations and definitions are similar to those in [3] and we indicate them here. Let $S$ denote the set of real numbers and $R$ a complete normed algebra over the real field with norm $|\cdot|$ and unity 1 such that $|1|=1$. The algebra of real numbers will be considered as a subalgebra of $R$. A function $B$ from $S \times S$ into $R$ is said to be order-additive (respectively, order-multiplicative) if $(x, z)$ is in $S \times S$ and $x \leqq y \leqq z$ or $x \geqq y \geqq z$, then $B(x, y)+B(y, z)=B(x, z)$ (respectively, $B(x, y) \cdot B(y, z)=B(x, z))$. Let $\mathrm{OA}^{+}$be the set of all order-additive functions from $S \times S$ into the nonnegative real numbers and $\mathrm{OM}^{+}$be the set of all order-multiplicative functions from $S \times S$ into the real numbers not less than one. Let $O A$ be the set of all order-additive functions $V$ from $S \times S$ into $R$ for which there is an $\alpha$ in $\mathrm{OA}^{+}$such that $|V| \leqq \alpha$, and let $\mathrm{OM}$ be the set of all order-multiplicative functions $W$ from $S \times S$ into $R$ for which there is a $\nu$ in $\mathrm{OM}^{+}$such that $|W-1|$ $\leqq \nu-1$. The set $\mathrm{OB}$ shall consist of all functions $F$ from $S$ into $R$ such that $d F$ is in OA-where $d F(x, y)$ is defined to be $F(y)-F(x)$. Note that $F$ is in $\mathrm{OB}$ if and only if $F$ is of bounded variation on each bounded subinterval of $S$.

If $(a, b)$ is in $S \times S$, then $\left(t_{p}\right)_{o}^{n}$ is a subdivision of $(a, b)$ if $t_{0}=a, t_{n}=b$ and $t_{p-1} \leqq t_{p}$ or $t_{p-1} \geqq t_{p}$ according to whether $a \leqq b$ or $a>b$. If $h$ is a function from $S \times S$ to $R$, then ${ }_{a} \sum^{b} h \sim \sum_{t} h=\sum_{1}^{n} h\left(t_{p-1}, t_{p}\right)$ and ${ }_{a} \prod^{b} h \sim \prod_{t} h=\prod_{1}^{n} h\left(t_{p-1}, t_{p}\right)$ and the limit is in the sense of refinements of subdivisions. For each $\alpha$ in $\mathrm{OA}^{+}, V$ in $\mathrm{OA}$ and $(a, b)$ in $S \times S$ let $\varepsilon^{+}[\alpha](a, b)={ }_{a} \Pi^{b}[1+\alpha]$ and $\varepsilon[V](a, b)={ }_{a} \Pi^{b}[1+V]$. Mac-

Received by the editors March 28, 1969. 
Nerney [3, Theorems 2.2,3.3, and 4.2] shows that $\mathcal{E}^{+}$is a bijection from $\mathrm{OA}^{+}$into $\mathrm{OM}^{+}, \varepsilon$ is a bijection from $\mathrm{OA}$ to $\mathrm{OM}$ and for each $P$ in $R$ and $a$ in $S$ there is a unique $U$ in $\mathrm{OB}$ such that

$$
U(z)=P+(R) \int_{z}^{a} V \cdot U
$$

for each $z$ in $S$. Furthermore, $U(z)=\varepsilon[V](z, a) \cdot P$. (R) $\int_{z}^{a} V \cdot U$ is approximated by $\sum_{1}^{n} V\left(t_{p-1}, t_{p}\right) \cdot U\left(t_{p}\right)$ where $\left(t_{p}\right)^{n}$ is a subdivision of $(z, a)$.

Using the techniques of Coppel $[1$, p. 3 ] one can show that for each $A$ in $R$,

$$
\mu[A]=\lim _{h \rightarrow+0} \frac{|1+h A|-1}{h}
$$

exists. Furthermore, (see $[1$, p. 41$]$ and references cited there) if $A$ and $B$ are in $R$, then $\mu[r A]=r \mu[A]$ for each positive real number $r$, $|\mu[A]| \leqq|A|, \mu[A+B] \leqq \mu[A]+\mu[B]$ and $|\mu[A]-\mu[B]| \leqq|A-B|$.

Definition. For each $A$ in $R$ let $\exp (A)=\sum_{0}^{\infty} A^{p} /(p !)$.

Lemma 1. If $A$ is in $R$, then

(i) $\mu[A]=\lim _{h \rightarrow+0}(|\exp (h A)|-1) / h$,

(ii) $|\exp (A)| \leqq \exp (\mu[A])$, and

(iii) $1+h \mu[A] \leqq|1+h A| \leqq 1+h \mu[A]+2(\exp (h|A|)-1-h|A|)$ for each $h \geqq 0$. In particular, if $2 h|A| \leqq 1$,

$$
|1+h A| \leqq 1+h \mu[A]+4 h^{2}|A|^{2} .
$$

INDICATION OF PROOF. If $2 h|A| \leqq 1$, then $|\exp (h A)-1-h A|$ $\leqq 2 h^{2}|A|^{2}$ and (i) follows immediately. By (i), for each $\epsilon>0$ there is an integer $n \geqq 1$ such that $|(|\exp (A / n)|-1) n-\mu[A]|<\epsilon$. Hence,

$$
\begin{aligned}
|\exp (A)| & \leqq|\exp (A / n)|^{n}=[1+((|\exp (A / n)|-1) n) / n]^{n} \\
& \leqq[1+(\mu[A]+\epsilon) / n]^{n} \leqq \exp (\mu[A])+\epsilon \cdot \exp (2|\mu[A]|+\epsilon)
\end{aligned}
$$

and (ii) follows. If $0<k<h$, then $(|1+k A|-1) / k \leqq(|1+h A|-1) / h$ which shows that $1+h \mu[A] \leqq|1+h A|$. Furthermore, if $h \geqq 0$, then $\mu[h A]=h \mu[A] \leqq h|A|$ so

$$
\begin{aligned}
|1+h A| & \leqq|\exp (h A)|+\sum_{2}^{\infty} h^{p}|A|^{p} /(p !) \\
& \leqq \exp (\mu[h A])+(\exp (h|A|)-1-h|A|)
\end{aligned}
$$

and (iii) follows. 
Lemma 2. Suppose that $V$ is in $\mathrm{OA}, \alpha$ is in $\mathrm{OA}^{+}$such that $|V| \leqq \alpha$, and $(a, b)$ is in $S \times S$. Then whenever $t$ is a subdivision of $(a, b)$ and $s$ is a refinement of $t, \sum_{t} \mu[V] \leqq \sum_{s} \mu[V] \leqq \alpha(a, b)$, so that ${ }_{a} \sum^{b} \mu[V]$ exists.

Lemma 3. Let $V$ be in $O A,(a, b)$ in $S \times S$ and $\alpha$ in $O A^{+}$such that $|V| \leqq \alpha$, then

(i) if there is a subdivision $\left(t_{p}\right)_{o}^{n}$ of $(a, b)$ such that $\alpha\left(t_{p-1}, t_{p}\right) \leqq \delta \leqq 1 / 2$ for each $1 \leqq p \leqq n$, then

$$
\left|\sum_{t}(|1+V|-1)-\sum_{t}[V]\right| \leqq 4 \delta \alpha(a, b),
$$

(ii) for each $\delta>0$ there is a subdivision $\left(u_{p}\right)_{o}^{n}$ of $(a, b)$ such that if $u_{p-1}<x \leqq y<u_{p}$ or $u_{p-1}>x \geqq y>u_{p}$, then $\alpha(x, y) \leqq \delta$, and

(iii) for each $x$ in $S$ and each $\epsilon>0$, there is a $\delta>0$ such that if $x<y$ $\leqq x+\delta$, then $\alpha(y, x+\delta) \leqq \epsilon$, and if $x>y \geqq x-\delta$, then $\alpha(x-\delta, y) \leqq \epsilon$.

INDICATION OF PROOF. By using part (iii) of Lemma 1 ,

$$
\left|\sum_{t}(|1+V|-1)-\sum_{t} \mu[V]\right| \leqq \sum_{1}^{n} 4\left|V\left(t_{p-1}, t_{p}\right)\right|^{2}
$$

and (i) follows. Both (ii) and (iii) are true since $\alpha$ can be uniformly approximated by step functions on bounded subintervals of $S$.

Theorem 1. Suppose that $V$ is in $O A$ and $(a, b)$ is in $S \times S$, then $\sum^{b}(|1+V|-1)$ exists.

IndiCATION OF PROOF. Suppose that $a<b, \epsilon$ is a positive number less than $1 / 2$, and $\alpha$ is in $\mathrm{OA}^{+}$such that $|V| \leqq \alpha$. By (ii) and (iii) of Lemma 3 , there is a subdivision $\left(u_{p}\right)_{0}^{n}$ of $(a, b)$ and sequences $\left(x_{p}\right)_{1}^{n}$ and $\left(y_{p}\right)_{1}^{n}$ so that if $1 \leqq p \leqq n$,

1. $u_{p-1}<x_{p}<y_{p}<u_{p}$ and $\alpha\left(x_{p}, y_{p}\right) \leqq \epsilon$,

2. if $u_{p-1}<z \leqq x_{p}$, then $\alpha\left(z, x_{p}\right) \leqq \epsilon / n$, and

3. if $y_{p} \leqq z<u_{p}$, then $\alpha\left(y_{p}, z\right) \leqq \epsilon / n$.

By Lemma 2, for each $1 \leqq p \leqq n$, there is a subdivision $s^{p}$ of $\left(x_{p}, y_{p}\right)$ such that if $t$ is a refinement of $s^{p}$, then $\left|\sum_{t} \mu[V]-\sum_{s^{p}} \mu[V]\right| \leqq \epsilon / n$. Let $(s(q))_{0}^{m}$ be a subdivision of $(a, b)$ whose range equals the union of the range of $u$ and the range of $s^{p}$ for each $1 \leqq p \leqq n$. We see that

$$
\begin{aligned}
\sum_{s}(|1+V|-1) & =\sum_{1}^{n}\left\{\left|1+V\left(u_{p-1}, x_{p}\right)\right|-1\right. \\
& \left.+\sum_{s^{p}}(|1+V|-1)+\left|1+V\left(y_{p}, u_{p}\right)\right|-1\right\} .
\end{aligned}
$$


Suppose that $\left(t_{q}\right)_{0}^{1}$ is a refinement of $s$. We can estimate $\left|\sum_{t}(|1+V|-1)-\sum_{s}(|1+V|-1)\right|$ by comparing the differences in each of the intervals $\left[u_{p-1}, x_{p}\right],\left[x_{p}, y_{p}\right]$ and $\left[y_{p}, u_{p}\right]$ for $1 \leqq p \leqq n$. Let $1 \leqq p \leqq n$ and suppose that $q_{1}$ and $q_{2}$ are integers such that $u_{p-1}$ $=t\left(q_{1}\right)<t\left(q_{1}+1\right)$ and $t\left(q_{2}\right)=x_{p}$. In the interval $\left[u_{p-1}, x_{p}\right]$ we have the estimate

$$
\begin{aligned}
& \left|\sum_{q_{1}+1}^{q_{2}}[|1+V(t(k-1), t(k))|-1]-\left(\left|1+V\left(u_{p-1}, x_{p}\right)\right|-1\right)\right| \\
& \quad \leqq\left|1+V\left(u_{p-1}, t\left(q_{1}+1\right)\right)\right|-\left|1+V\left(u_{p-1}, x_{p}\right)\right|+\alpha\left(t\left(q_{1}+1\right), x_{p}\right) \\
& \quad \leqq 2 \alpha\left(t\left(q_{1}+1\right), x_{p}\right) \\
& \quad \leqq 2 \epsilon / n .
\end{aligned}
$$

A similar estimate holds in the interval $\left[y_{p}, u_{p}\right]$. Now suppose $q_{1}$ and $q_{2}$ are integers such that $t\left(q_{1}\right)=x_{p}$ and $t\left(q_{2}\right)=y_{p}$ and let $r$ be the subdivision of $\left(x_{p}, y_{p}\right)$ such that $r(k)=t\left(q_{1}+k\right)$ for each $0 \leqq k \leqq q_{2}-q_{1}$. By using part (i) of Lemma 3, we have

$$
\begin{aligned}
\mid \sum_{r}(\mid 1 & +V \mid-1)-\sum_{s^{p}}(|1+V|-1) \mid \\
\leqq & \left|\sum_{r}(|1+V|-1)-\sum_{r} \mu[V]\right|+\left|\sum_{r} \mu[V]-\sum_{s^{p}} \mu[V]\right| \\
& \quad+\left|\sum_{s^{p}} \mu[V]-\sum_{s^{p}}(|1+V|-1)\right| \\
\leqq & 4 \epsilon \alpha\left(x_{p}, y_{p}\right)+\epsilon / n+4 \epsilon \alpha\left(x_{p}, y_{p}\right) \\
\leqq & \leqq \\
&
\end{aligned}
$$

Combining the above estimates shows that

$$
\left|\sum_{t}(|1+V|-1)-\sum_{s}(|1+V|-1)\right| \leqq(5+8 \alpha(a, b)) \cdot \epsilon
$$

and the assertion of the theorem follows.

If $V$ is in OA the function $\gamma[V]$ from $S \times S$ into $R$ defined by $\gamma[V](x, y)={ }_{x} \sum^{y}(|1+V|-1)$ is clearly order-additive. The inequality $|(|1+V(x, y)|-1)| \leqq|V(x, y)|$ shows that $\gamma[V]$ is in OA and if $\alpha$ is in $\mathrm{OA}^{+}$such that $|V| \leqq \alpha$, then $|\gamma[V]| \leqq \alpha$.

Let OAI denote the set of all $V$ in OA such that for each $x$ in $S$, $1+V(x, x+), 1+V(x+, x), 1+V(x-, x)$ and $1+V(x, x-)$ have multiplicative inverses in $R$. J. V. Herod [2] shows that $\varepsilon[V](a, b)$ has a multiplicative inverse in $R$ for each $(a, b)$ in $S \times S$ if and only 
if $V$ is in OAI. Furthermore, there is a bijection $G$ from OAI in to OAI so that each of the following holds.

(1) $G(V)(a, b)=-{ }_{b} \sum^{a}(1+V)^{-1} V$ for each $(a, b)$ in $S \times S$,

(2) $G(V)(a, b)=-V(b, a)$ for each $(a, b)$ in $S \times S$ if and only if ${ }_{a} \sum^{b}\left|V^{2}\right|=0$ for each $(a, b)$ in $S \times S$, and

(3) $\varepsilon[G(V)](a, b) \cdot \varepsilon[V](b, a)=1$ for each $(a, b)$ in $S \times S$.

Lemma 4. If $V$ is in $O A,(a, b)$ is in $S \times S$ and $\epsilon$ is a positive number, there is a subdivision $u$ of $(a, b)$ such that whenever $t$ is a refinement of $u$, $\sum_{t}|\gamma[V]-(|1+V|-1)| \leqq \epsilon$.

TheOREM 2. Suppose that $V$ is in $O A$ and $\alpha$ is in $O A^{+}$so that $|V| \leqq \alpha$. Then for each $(a, b)$ in $S \times S$

(i) $|\varepsilon[V](a, b)| \leqq \varepsilon[\gamma[V]](a, b) \leqq \mathcal{E}^{+}[\alpha](a, b)$.

If, in addition, $V$ is in OAI and $\beta$ is in $\mathrm{OA}^{+}$so that $|\gamma[G(V)]| \leqq \beta$, then

(ii) $\varepsilon[V](a, b) \mid \geqq\{\varepsilon[\gamma[G(V)]](b, a)\}^{-1} \geqq\left\{\varepsilon^{+}[\beta](b, a)\right\}^{-1}$.

Remarks. Compare with Coppel [1, p. 58]. Also, it can be shown that, with the supposition of Theorem $2,|\gamma[G(V)]| \leqq G(\alpha)$.

INDICATION OF PROOF. If $\epsilon$ is a positive number there is a subdivision $t$ of $(a, b)$ such that

(1) $|\varepsilon[V](a, b)| \leqq\left|\prod_{t}(1+V)\right|+\epsilon$,

(2) $\varepsilon[\gamma[V]](a, b) \leqq \prod_{i}(1+\gamma[V])+\epsilon$, and

(3) $\sum_{t}|\gamma[V]-(|1+V|-1)| \leqq \epsilon$.

Hence,

$$
|\varepsilon[V](a, b)| \leqq \prod_{1}^{n}\left(1+\gamma[V]\left(t_{p-1}, t_{p}\right)+s_{p}\right)+\epsilon
$$

where $s_{p}=\left|1+V\left(t_{p-1}, t_{p}\right)\right|-1-\gamma[V]\left(t_{p-1}, t_{p}\right)$. Since

$$
\begin{aligned}
\prod_{1}^{n} & \left(1+\gamma[V]\left(t_{p-1}, t_{p}\right)+s_{p}\right)-\prod_{1}^{n}\left(1+\gamma[V]\left(t_{p-1}, t_{p}\right)\right) \\
= & \sum_{1}^{n}\left[\prod_{1}^{p-1}\left(1+\gamma[V]\left(t_{q-1}, t_{q}\right)\right] \cdot s_{p} \cdot\left[\prod_{p+1}^{n}\left(1+\gamma[V]\left(t_{q-1}, t_{q}\right)+s_{p}\right)\right]\right.
\end{aligned}
$$

and $\sum_{1}^{n}\left|s_{p}\right| \leqq \epsilon$, it follows that

$$
|\varepsilon[V](a, b)| \leqq \varepsilon[\gamma[V]](a, b)+\epsilon \cdot(2+\exp (2 \alpha(a, b)+\epsilon)) .
$$

Part (i) now follows. If $V$ is in OAI, $\varepsilon[G(V)](b, a) \cdot \varepsilon[V](a, b)=1$ and it follows that $|\varepsilon[V](a, b)| \geqq|\varepsilon[G(V)](b, a)|^{-1}$. The assertion of the theorem follows by applying part (i) to $G(V)$. 
Example. Let $a$ be in $S, f$ be a continuous function from $S$ into $R$ and let $F(z)=\int_{z}^{a} f(x) d x$ for each $z$ in $S$. Part (iii) of Lemma 1 shows that $(|1+h f|-1) / h$ tends to $\mu[f]$ uniformly on bounded subintervals of $S$. If $(a, b)$ is in $S \times S$ and $t$ is a subdivision of $(a, b)$, then estimates of the type

$$
\begin{aligned}
\gamma[d F](a, b) & \sim \sum_{1}^{n}\left(\left|1+\int_{t_{p}}^{t_{p-1}} f(x) d x\right|-1\right) \\
& \sim \sum_{1}^{n} \frac{\left|1+\left(t_{p-1}-t_{p}\right) f\left(t_{p-1}\right)\right|-1}{\left(t_{p-1}-t_{p}\right)}\left(t_{p-1}-t_{p}\right)
\end{aligned}
$$

show that

$$
\gamma[d F](a, b)=-\int_{b}^{a} \mu[-f(x)] d x \quad \text { if } a \leqq b,
$$

and

$$
\gamma[d F](a, b)=\int_{b}^{a} \mu[f(x)] d x \quad \text { if } a \geqq b .
$$

Hence, if $P$ is in $R$ and $U$ is in OB such that $U(z)=\varepsilon[d F](z, a) \cdot P$ for each $z$ in $S$, part (i) of Theorem 2 shows that

$$
\begin{aligned}
& |U(z)| \leqq|P| \exp \left(\int_{a}^{z} \mu[f(x)] d x\right) \quad \text { if } z \geqq a, \\
& \leqq|P| \exp \left(-\int_{a}^{z} \mu[-f(x)] d x\right) \quad \text { if } z \leqq a .
\end{aligned}
$$

Since $d F$ is in OAI and ${ }_{x} \sum^{y}\left|d F^{2}\right|=0$ for each $(x, y)$ in $S \times S$, $G(V)(x, y)=-d F(y, x)$ and part (ii) of Theorem 2 shows that

$$
\begin{aligned}
|U(z)| & \geqq|P| \exp \left(-\int_{a}^{z} \mu[-f(x)] d x\right) & \text { if } z \geqq a, \\
& \geqq|P| \exp \left(\int_{a}^{z} \mu[f(x)] d x\right) & \text { if } z \leqq a .
\end{aligned}
$$

Consequently, Theorem 2 contains Theorem 3 of $[1$, p. 58]. 


\section{REFERENCES}

1. W. A. Coppel, Stability and asymptotic behavior of differential equations, Heath, Boston, 1965.

2. J. V. Herod, Multiplicative inverses of solutions for Volterra-Stielties integral equations, Proc. Amer. Math. Soc. 22 (1969), 650-656.

3. J. S. MacNerney, Integral equations and semigroups, Illinois J. Math. 7 (1963), 148-173.

4. - - A linear initial value problem, Bull. Amer. Math. Soc. 69 (1963), 314329.

Georgia Institute of Technology 